\title{
Hypothesen
}

\section{Zur Immunpathogenese des Morbus Crohn}

\author{
W. Stöcker, M. Otte und P. C. Scriba \\ Klinik für Innerc Medizin (Dircktor: Prof. Dr. P. C. Scriba) der Medizinischen Hochschule Lübeck
}

Patienten mit Morbus Crohn essen überdurchschnittlich viel Zucker $(18,20,21,25,26)$ und Stärke (21). Demgegenüber ist der Zuckerkonsum bei Patienten mit Colitis ulcerosa normal $(5,36)$. Stärke hat keine stimulierende Wirkung auf das exokrine Pankreas (37), und auch Glucose steigert die Pankreassekretion nicht $(14,35,37)$ oder nur kurzfristig (15). In den Dünndarm infundierte Glucose hemmt sogar die sekretin- und pankreozyminstimulierte Pankreassekretion (10).

Die Ernährungsweise der Patienten führt also offenbar zu einer im Vergleich mit Gesunden geringeren Pankreassekretion und deutet deshalb darauf hin, daß beim $\mathrm{M}$. Crohn eine Unverträglichkeit gegen Pankreassekret vorliegen könnte. Dieser Verdacht wird durch den Nachweis einer für den M. Crohn spezifischen Autoimmunität gegen exokrines Pankreas (34) bestätigt. Wir glauben daher, jetzt einige Fragen zur Pathogenese des M. Crohn beantworten zu können.

\section{Hypothese}

Der Morbus Crohn ist eine Autoimmunerkrankung. Die Entzündung des Darmes wird durch Immunreaktionen gegen Bestandteile des Pankreassekrets hervorgerufen. Unbewußt versuchen die Patienten, durch eine geeignete Ernährung ihr exokrines Pankreas ruhigzustellen, um die Belastung des Darmes mit dem Antigen zu reduzieren.

Wodurch die Autoimmunität beim M. Crohn ausgelöst wird, ist nicht bekannt. Vom Zeitpunkt der Sensibilisierung an könnte die Erkrankung nach folgendem Schema ablaufen: Antigene des Pankreassekrets gelangen über den Ductus pancreaticus in das Darmlumen, diffundieren in die Darmwand und reagieren hier mit Autoantikörpern. Dabei entstehen Immunkomplexe, die sich in Mucosa und Submucosa ablagern und das Komplementsystem aktivieren. Mediatoren locken Granulozyten an, und deren Enzyme zerstören das Gewebe.

Vielleicht breiten sich die Antigene, vom Pankreas ausgehend, auch hämatogen aus, und sie präzipitieren im Bereich der Kapillaren mit den in der befallenen Darm-

Dtsch. med. Wschr. 109 (1984), 1984-1986

(C) 1984 Georg Thieme Verlag Stuttgart · New York wand in hohen Konzentrationen vorliegenden Autoantikörpern. Alternativ könnten sich die Antigene an die Enterozyten oder an andere Strukturen der Darmwand binden, die dadurch zu Zielen der humoralen und zellulären Autoimmunität würden.

Die extraintestinalen Manifestationen des M. Crohn könnten zum Teil durch Ablagerungen von Immunkomplexen aus Pankreasantigenen und Autoantikörpern hervorgerufen werden, zum Teil auch durch eine Mitreaktion von Organen, die ähnliche oder die gleichen Antigene besitzen wie das exokrine Pankreas.

\section{Begründung}

Beim M. Crohn kann man in den befallenen Darmabschnitten histologische Zeichen einer Immunreaktion erkennen: Die Darmwand ist mit Lymphozyten, Plasmazellen und Granulozyten infiltriert. Regelmäßig sind zahlreiche Lymphfollikel mit und ohne Keimzentren anzutreffen $(12,28)$. Es werden zwölfmal so viele Plasmazellen gefunden (in schwer entzündeten Bezirken mit noch erhaltener Schleimhaut) wie bei Gesunden, die Zahl der IgG-produzierenden Plasmazellen ist sogar um den Faktor 60 gesteigert $(4,6)$. Bisher war nicht bekannt, gegen welche Antigene sich diese Abwehrfront richtet.

Das Darmlumen der Crohn-Patienten scheint toxische Substanzen zu enthalten: Eine akute Colitis granulomatosa bessert sich in den meisten Fällen sehr schnell, wenn man ein Ileostoma oder ein Kolostoma anlegt und dadurch das Kolon ausschaltet $(17,22)$. Bei Colitis ulcerosa hilft diese Behandlung nicht (17). Für die Annahme toxischer Substanzen im Darmlumen spricht auch die Beobachtung, daß sich durch eine intestinale Lavage mit physiologischer Kochsalzlösung bei einigen CrohnPatienten der Aktivitätsindex verringern ließ (38).

Die toxischen Substanzen könnten aus dem Pankreas stammen. Hinweise dafür sind das spontane Ernährungsverhalten der Crohn-Patienten, das möglicherweise zu einer Reduktion der Pankreassekretion führt, ferner der Therapieerfolg mit parenteraler Ernährung (2), die das exokrine Pankreas ruhigstellt (33), und schließlich die häufige Besserung des M. Crohn durch eine Elementardiät $(27,29)$, bei der das Pankrcas wegen der schnellen Resorption der Nährstoffe wahrscheinlich nicht nachhaltig stimuliert wird. 
Bei 39\% der Patienten mit M. Crohn wurden im Serum Autoantikörper gegen exokrines Pankreas nachgewiesen (34). Während niedrige Konzentrationen dieser Autoantikörper bei Pankreatitis vorkommen können, gelegentlich auch bei Colitis ulcerosa und bei Gesunden, wurden hohe Konzentrationen (Titer $1: 100$ oder höher) ausschließlich beim M. Crohn gefunden.

Die im indirekten Immunfluoreszenztest feststellbare Verteilung der Antigene im Pankreasgewebe (34) und Absorptionsversuche mit Pankreassaft (13) zeigen, daß die Autoantikörper gegen Sekretionsprodukte des Pankreas gerichtet sind.

Außer den histologischen Veränderungen im befallenen Gewebe und dem Auftreten spezifischer Autoantikörper im Serum hat die Crohnsche Erkrankung weitere Gemeinsamkeiten mit gesicherten Autoimmunkrankheiten: Sie verläuft chronisch und in Schüben, sie spricht auf immunsuppressive Therapie an, das weibliche Geschlecht ist stärker betroffen, und die Krankheit tritt familiär gehäuft auf. Eine Korrelation zu einem bestimmten Antigeñ des HLA-Systems (3: HLA-A 9 selten, 32: HLA-B 12 häufig) wird allerdings von den meisten Untersuchern bestritten $(8,11,24,30)$.

\section{Diskussion}

Viele Patienten mit M. Crohn geben an, ihre Vorliebe für Süßigkeiten habe schon vor der Erkrankung bestanden (25). Das muß nicht bedeuten, daß die Krankheit durch Zucker oder durch in den Süßigkeiten enthaltene Begleitstoffe verursacht wird, weil man nicht weiß, zu welchem Zeitpunkt sie ausgelöst wurde: Sie könnte lange vor den ersten Symptomen begonnen haben. Außerdem waren unter den befragten Patienten viele mit langjähriger Krankheitsanamnese (25), und es wird angezweifelt, daß sie sich noch ausreichend genau an ihre Ernährungsgewohnheiten vor dem Auftreten der Erkrankung erinnern können. In den meisten Fällen ist der Zuckerverbrauch innerhalb eines halben Jahres nach dem Bekanntwerden der Diagnose noch normal (20). Offenbar stellen sich die Patienten in dieser Zeit darauf ein, daß ihr Intestinaltrakt durch Pankreassekret geschädigt wird. Wahrscheinlich meiden sie dann unbewußt Proteine und Fette, und das Energiedefizit kompensieren sie durch Zucker und Stärke. Dadurch erreichen sie, daß ihr Pankreas weniger Sekret produziert.

Nach dem Zweiten Weltkrieg ist der M. Crohn in den wohlhabenden Ländern häufiger geworden. Auch dieses Phänomen ließe sich mit der vorliegenden Hypothese erklären: Eine reichliche Ernährung mit einem hohen Anteil an Proteinen und Fetten stimuliert das exokrine Pankreas stark und langdauernd. Im Vergleich zur Situation bei einem geringen Nahrungsangebot wird dadurch das Immunsystem des Darms mit mehr Pankreassekret konfrontiert, und die Wahrscheinlichkeit, sich zu sensibilisieren, wird höher. Nach Guthy (16) tritt der M. Crohn in Ländern mit hohem Margarineverbrauch besonders häufig auf. Man sollte deshalb überprüfen, ob Margarine die Pankreassekretion stärker anregt als Butter.

Eine klinisch manifeste Pankreatitis ist beim M. Crohn selten, unabhängig davon, ob Autoantikörper gegen exokrines Pankreas vorliegen oder nicht (34). Aber die exokrine Pankreasfunktion ist beim $M$. Crohn eingeschränkt (19). Das wurde als Folge einer nicht näher definierten "Begleit-Pankreatitis" (19) oder einer Mangelernährung $(9,19)$ angesehen. Bei 15 von 39 Crohn-Patienten wurden fibrotische Veränderungen des exokrinen Pankreas nachgewiesen, keiner dieser Patienten hatte eine klinisch manifeste Pankreaserkrankung (7). Nur ausnahmsweise kommt es beim M. Crohn zu einer akuten Pankreatitis, sie wird auf eine Abflußbehinderung des Sekrets bei Mitbeteiligung des Duodenums zurückgeführt, aber nicht auf eine Immunreaktion (1, 23). Bei Wistar-Ratten entsteht eine chronische Pankreatitis, wenn man sie mit homogenisiertem Pankreasgewebe immunisiert oder wenn man ihnen das Serum immunisierter Kaninchen mehrmals parenteral verabreicht (31). Weshalb bekommen die Tiere eine Pankreatitis und keine Enterokolitis, und weshalb steht beim M. Crohn nicht eine Pankreaserkrankung im Vordergrund?

Die Injektion des Pankreashomogenats führt bei den Tieren zu einer Immunität gegen viele im Pankreas enthaltene Antigene (31), darunter sicherlich auch gegen Zellmembranantigene. Diese sind dem Immunsystem zugänglich, sie können unmittelbar im Pankreas attakkiert werden, und auch die entzündlichen Folgereaktionen laufen im Pankreas ab. Eine dem M. Crohn ähnliche Darmentzündung wurde bei den Tieren möglicherweise deshalb nicht ausgelöst, weil das für die Immunisierung verwendete Pankreashomogenat nicht genügend Sekret enthielt.

Die Autoantikörper beim M. Crohn sind nicht gegen das ganze Spektrum der Pankreasantigene gerichtet, sondern nur gegen Bestandteile des Pankreassekrets (13). Das von den Acinuszellen gebildete Sekret wird über das Pankreasgangsystem in das Duodenum abgegeben, und es kommt im Pankreas mit den immunkompetenten Zellen wahrscheinlich nicht in Kontakt. Daher entwickelt sich auch keine klinisch auffallende Entzündung des Pankreas. Erst im Darm setzt sich das Immunsystem mit den Antigenen auseinander.

Warum aus dem Darm der Crohn-Patienten bestimmte Bakterien und Viren besonders häufig isoliert werden können, läßt sich bislang nicht erklären. Von einigen Autoren wird der M. Crohn sogar als Infektionskrankheit angesehen (Übersicht: 39). Vielleicht sind die gefundenen Erreger Ausdruck einer erhöhten Infektanfälligkeit der geschädigten Schleimhaut, vielleicht spielen sie aber auch bei der Auslösung der Autoimmunität eine Rolle, deren Mechanismus beim M. Crohn, wie bei allen gesicherten Autoimmunerkrankungen, bisher nicht durchschaut wird.

Die Hypothese löst noch nicht alle Rätsel des Morbus Crohn, sie bietet aber ein auf vielen gesicherten Erkennt- 
nissen beruhendes Konzept, das durch weitere Untersuchungen nachzuprüfen ist und das zu neuen Möglichkeiten für die Prophylaxe und die Therapie des Morbus Crohn führen könnte.

\section{Literatur}

(1) Altman, H. S., G. Phillips, S. Bank, H. Klotz: Pancreatitis associated with duodenal Croln's disease. Amer. J. Gastroent. $78(1983), 174$

(2) Anderson, D. L., H. W. Boyce jr.: Use of parenteral nutrition in treatment of advanced regional enteritis. Dig. Dis. 18 advanced region
(1973), 633

(3) Asquith, P., P. Mackintosh, P. L. Stokes, G. K. T. Holmes, W. T. Cooke: Histocompatibility antigens in patients with inflammatory bowel disease. Lance 1974/I, 113.

(4) Baklien, K., P. Brandtzaeg: Immunohistochemical characterisation of local immunoglobulin formation in Crohn's discase of the ileum. Scand. J. Gastroent. 11 (1976), 447.

(5) Brandes J. W., A. Stenner, G. A. Mar tini: Ernährungsgewohnheiten der $\mathrm{Pa}$ tienten mit Colitis ulcerosa. Z. Gastroent. 17 (1979), 834

(6) Brandtzaeg, P., K. Baklien: Immunopathology of the intestinal lesion in Crohn's diseasc. Z. Gastroent. 17 (1979), Suppl. 77.

(7) Chapin, L. E., H. H. Scudamorc, A. H. Baggenstoss, J. A. Bargen: Regional enteritis: associated visceral changes. Gastroenterology 30 (1956), 404.

(8) Delpre, G., U. Kadish, E. Gazit, H Joshua, R. Zamir: HLA antigens in ulceracive colitis and Crohn's disease in Isracl. Gastroentcrology 78 (1980), 1452.

(9) Drciling, D. A.: The role of minor pancreatic exocrine deficiency in digestive disorders. A review. Amer. J. Gastroent. 78 (1983), 732

(10) Dyck, W. P.: Influence of intrajcjunal glucose on pancreatic exocrine function in man. Gastrocnterology 60 (1971), 864
(11) Eade, O. E., C. Moulton, B. R. MacPherson, S. St. Andre-Ukena, R. J. Albertini, W. L. Becken: Discordant HLA haplotype segregation in familial Crohn's disease. Gastroenterology 79 (1980) 271

(12) Fahrländer, H., L. Bianchi, M. J. Mihatsch: Die chronisch entzündlichen Harnack, G. A. Martini, A. Prader, R. Schoen, H. P. Wolff (Hrsg.): Ergebnisse der Inneren Medizin und Kinderheilkunde, Bd. 42 (Springer: Berlin-HeidelbergNew York 1979), 1.

(13) Finkbeiner, H., W. Stöcker, M. Otte: in Vorbereitung.

(14) Forell, M. M., H. Stahlheber, P. Lehnert, W. Londong, H. Teufel, $H$. Fritz, O. Roder: Physiologic des exokrinen Pankreas. In Forell, M. M. (Hrsg.): Handbuch der Inneren Medizin, 5. Auf1. Bd. 3, Verdauungsorgane, Teil 6, Pankreas (Springer: Berlin-Heidelberg-New York 1976).

(15) Go, V. L. W., A. F. Hofmann, W. H. J. Summerskill: Pancreozymin bioassay in man based on pancreatic enzyme secre tion. Potency of specific amino acids and other digestive products. J. clin. Invest. $49(1970), 1558$

(16) Guthy, E.: Morbus Crohn und Nahrungsfette. Hypothese zur Ätiologie der Enteritis regionalis. Dtsch. med. Wschr. 107 (1982), 71

(17) Harper, P. H., S. C. Truelove, E. C. G. Lee, M. G. W. Kettlewell, D. P. Jewell: Split ileostomy and ileocolostomy for Crohn's discase of the colon and ulcerative colitis. A 20 year survey. Gut 24 (1983), 106.

(18) Heaton, K. W. J. R. Thornton, P. $M$. Emmet: Dietary factors in Crohn's disease. Z. Gastroent. 17 (1979), Suppl. 140 . Darmkrankheiten. In Frick, P., G.-A. von
(19) Hoppe-Seyler, P., D. Holtermann W. Gerok: Untersuchungen der exokrinen Pankreasfunktion bei M. Crohn. Z Gastroent. 19 (1981), 570.

(20) Järnerot, G., I. Järnmark, K. Nilsson: Consumption of refined sugar by patients with Crohn's disease, ulcerative colitis, or irritable bowel syndrome. Scand. J. Gastroent. 18 (1983), 999.

(21) Kasper, H., H. Sommer: Dietary fiber and nutrient intake in Crohn's disease. Amer. J. clin. Nutr. 32 (1979) 1898.

(22) Korelitz, B. I., L. J. Cheskin, N Sohn, S. C. Sommers: The nature of the proctitis occurring after diversion of the fecal stream in Crohn's disease. A new concept in management. Gastroenterol. ogy 84 (1983), 1214

(23) Legge, D. A., H. N. Hoffman, H. C. Carlson: Pancreatitis as a complication of regional enteritis of the duodenum. Gastroenterology 61 (1971), 834.

(24) Mallas, E. G., P. Mackintosh, P. Asquith, W. T. Cooke: Histocompatibility antigens in inflammatory bowel disease. Gut 17 (1976), 906

(25) Martini, G. A., J. W. Brandes: Increased consumption of refined carbohydrates in patients with Crohn's diseasc. Klin. Wschr. 54 (1976), 367.

(26) Miller, B., F. Fervers, R. Rohbeck, Strohmeyer: Zuckerkonsum bei Patienten mit Morbus Crohn. Verh. dtsch. Ges inn. Med. 82 (1976) 922.

(27) Morin, C. L., M. Roulet, C. C. Roy, A. Weber: Continuous elemental enteral alimentation in children with Crohn's disease and growth failure. Gastroenterology $79(1980), 1205$

(28) Morson, B. C. Pathology of Crohn's disease. Clin. Gastroent. 1 (1972), 265

(29) O'Morain, C. A., A. W. Segal, A. J. Levi: Elemental diets in the treatment of acute Crobn's disease. A controlled study. Gut 23 (1983), A 891.

Dr. W. Stöcker, Prof. Dr. M. Otte, Prof. Dr. P. C. Scriba

Klinik für Innere Medizin

Medizinische Hochschule

2400 Lübeck, Ratzeburger Allee 160
(30) Peña, A. S., L. Biemond, G. Kuiper, I. T. Weterman, A. van Leeuwen, I. Schreuder, J. J. van Rood: HLA antigen distribution and HLA haplotype segregation in Crohn's disease. Tissue Antigens 16 (1980), 56.

(31) Richter, K.: Isoimmunpankreatitis. Eine tierexperimentelle Studic. In Doer W., H. Leonhardt (Hrsg.); Normale und pathologische Anatomie. Bd. 46

(Thicme: Stuttgart-Ncw York 1983)

(32) Smolen, J. S., A. Gangl, P. Polterauer, E. J. Menzel, W. R. Mayr: HLA antigens in inflammatory bowcl diseasc. Gastroenterology 82 (1982), 34

(33) Stabile, B. E., M. Borzatta, R. S. Stubbs, H. T. Debas: Intravenous mixed amino acids and fats do not stimulate exocrine pancreatic secretion. Amer. J. Physiol. 246 (1984), G 274

(34) Stöcker, W., M. Otte, S. Ulrich, D. Normann, K. Stöcker, G. Jantschek: A toantikörper gegen exokrines Pankreas und gegen intestinale Becherzellen in de Diagnostik des Morbus Crohn und der Colitis ulkerosa. Dtsch, med. Wschr. 109 (1984), 1963.

(35) Sum, P. T., R. M. Preshaw: Intraduodenal glucose infusion and pancreatic secretion in man. Lancet 1967/II, 340.

(36) Thornton, J. R., P. M. Emmett, K. W. Heaton: Diet and ulcerative colitis. Brit. med. J. 1980/1, 293.

(37) Wang, C. C., M. I. Grossman: Physiological determination of relcase of secretin and pancrcozymin from intestine of dogs with transplanted pancreas. Amer. J. Physiol. 164 (1951), 527. (38) Wellmann, W., F. W. Schmidt: Intestinal lavage in the treatment of Crohn's disease. A pilot study. Klin. Wschr. 60 (1982), 371

(39) Whorwell, P. J.: Infectious agents in Crohn's disease - fact or artefact? Scand. J. Gastroent. 16 (1981), 161. 